\title{
Aterogênese em artéria ilíaca comum de suínos submetidos à homocisteinemia induzida pela ingestão de metionina
}

\author{
Atherogenesis in swine iliac artery with homocystinemia induced by methionine ingestion \\ Luís H enrique Gil França ${ }^{1}$, Adamastor H umberto Pereira², Sílvio C ésar Perini ${ }^{3}$, \\ Celso C urcio Aveline ${ }^{4}$, Rodrigo Argenta 3 , Roseli de 0 liveira M ollerke, M arcos Eugenio Soares, \\ Fernanda N óbrega ${ }^{6}$, M árcio Poletto Ferreira $^{6}$
}

\begin{abstract}
Resumo
0 bjetivo: Avaliar os efeitos da homocisteinemia induzida na artéria ilíaca de suínos.

M aterial e método: Realizou-se estudo experimental comparativo em dois grupos homogêneos de sete suínos da raça M acau, com peso entre 20 e $30 \mathrm{~kg}$ durante 30 dias. 0 sanimais foram divididos em dois grupos, sendo um deles al imentado com metionina adicionada à dieta por um período de 4 semanas. Foram colhidas amostras de sangue para a dosagem de colesterol, triglicerídeos, H D L ehomocisteína. O s animais foram submetidos à arteriografia para avaliação da perviedade das artérias ilíacas e, posteriormente, sacrificados. As arté rias ilíacas foram enviadas para análise histológica.

Resultados: 0 sanimais sobreviveram ao experimento, enão houve alterações significativas nos níveis de colesterol total, triglicerídeos e H D L nos dois grupos. 0 exame microscópico do grupo-controle não apresentou alterações patológicas e foi semel hante em todas as preparações examinadas. No grupo da dieta com metionina, as placas eram formadas por macrófagos espumosos, mas não foram observadas céIulas musculares lisas, cristais de colesterol ou células inflamatórias. A túnica média apresentava-se com lâmina elástica interna íntegra. No grupo-controle, não houve alteração nos níveis de homocisteína durante o experimento. $\mathrm{N}$ o grupo-metionina, houve aumento dos níveis séricos da homocisteína, com valor médio de 59,80 $\mu \mathrm{mol} / \mathrm{I}$ após 30 dias de dieta rica em metionina.

Conclusão: A homocisteinemia induzida pela metionina causa aterogênese nas artérias ilíacas de suínos.
\end{abstract}

Palavras-chave: H omocisteína, suínos, artéria ilíaca.

\begin{abstract}
0 bjective: To assess the effects of induced homocystinemia in the swine iliac artery.

M aterial and method: A comparative experimental study with two homogeneous groups of seven pigs from the $\mathrm{M}$ acao specimen, weighed between 20 and $30 \mathrm{~kg}$, which were assessed during 30 days. The pigs were divided into two groups. One of them was fed with a methionine-rich diet for a 4-week period. Blood samples were collected for analyses of total cholesterol, triglycerides, HDL and homocysteine concentrations. The animals were submitted to arteriography to evaluate the patency of iliac arteries and then sacrificed. Theiliac artery segment was removed for histological analysis.

Results: All animals survived the procedure, and there were no significant changes in total cholesterol, triglycerides and $\mathrm{HDL}$ concentrations in both groups. Microscopic examinations of the control group did not show pathological changes and was similar in all analyses. In the group receiving the methionine diet, the plaques were formed by foamy macrophages, but smooth muscle cells, cholesterol crystals or inflammatory cells were not seen. The tunica media had the internal elastic lamina intact. In the control group, there was no change in homocysteine levels during the experiment. In the methionine group, there was an increase in plasma homocysteine levels, with an average value of $59.80 \mu \mathrm{mol} / \mathrm{l}$ after 30 days with a methionine-rich diet.
\end{abstract}

Conclusion: Homocystinemia induced by methionine causes atherogenesis in the swine iliac artery.

Key words: H omocysteine, pigs, iliac artery.

\footnotetext{
1. Cirurgião vascular. Mestre em Clínica Cirúrgica, Universidade Federal do Paraná (UFPR), Curitiba, PR. Aluno de Doutorado em Cirurgia, Universidade Federal do Rio Grande do Sul (UFRGS), Porto Alegre, RS. Membro efetivo, Sociedade Brasileira de Angiologia e de Cirurgia Vascular.

2. Professor adjunto e Chefe do Serviço de Cirurgia Vascular, Hospital de Clínicas de Porto Alegre (HCPA), Porto Alegre, RS.

3. Cirurgião vascular. Aluno de Mestrado em Cirurgia, UFRGS, Porto Alegre, RS.

4. Anestesista, Hospital São Lucas, Pontifícia Universidade Católica do Rio Grande do Sul (PUCRS), Porto Alegre, RS.

5. Doutora. Professora, Faculdade de Veterinária, UFRGS, Porto Alegre, RS.

6. Faculdade de Veterinária, UFRGS, Porto Alegre, RS.
}

Este estudo foi realizado no Serviço de Cirurgia Vascular do HCPA, Programa de Pós-Graduação em Cirurgia, UFRGS, Porto Alegre, RS.

Artigo submetido em 19.02.06, aceito em 16.03.06. 
As doenças do aparelho circulatório apresentam altas taxas de morbidade e mortalidade, e, dentre as várias etiologias conhecidas, a mais comum é a aterosclerose. Atualmente, a aterosclerose é definida como um processo crônico, progressivo e sistêmico, conseqüente a uma resposta inflamatória e fibroproliferativa causada por agressão à superfície arterial. N os últimos anos, houve um considerável progresso nas pesquisas experimentaiseclínicas, que evidenciaram a importância do processo inflamatório na sua gênese ${ }^{1,2}$.

Estudos epidemiológicos revelam que pessoas com homocisteína elevada têm risco aumentado de serem acometidas de doença cardiovascular ${ }^{3}$. Estudosclínicos e experimentais sugerem que concentrações anormalmente elevadas de homocisteína podem ser responsáveis por tendências aterogênicas etrombóticas em pacientes com hiper-homocisteinemia, embora o mecanismo exato de sua ação ainda não tenha sido completamente elucidado. A importante questão de como a homocisteína- aminoácido quecontém enxofre - afeta os processos bioquímicos das células e tecidos das paredes arteriais para produzir placas ateroscleróticas é ainda hoje um campo ativo de investigação ${ }^{4}$.

0 presente trabalho propõe o desenvolvimento de um modelo experimental de lesão aterogênica em suínos, mediante a administração por via oral de metionina, um aminoácido do produto da homocisteína, para indução da lesão.

\section{0 bjetivo}

Diante da hipótese de que uma dieta rica em metionina induz a hiper-homocisteinemia em suínose, conseqüentemente, a aterogênese em artérias ilíacas destes animais, o objetivo desta pesquisa é desenvolver um modelo experimental de aterogênese em suínos e avaliar os efeitos da homocisteinemia induzida pela dieta com adição de metionina em artéria ilíaca de suínos.

\section{M aterial e método}

0 trabalho foi realizado na U nidadedeExperimentação Animal do C entro de Pesquisas do H ospital de Clínicas de Porto Alegre (H CPA), vinculado à U niversidade Federal do Rio G rande do Sul.

\section{Critérios de inclusão}

Foram incluídostodososanimaisquepreencheram os requisitos nos itens anteriores.

\section{Critérios de exclusão}

O s critérios de exclusão, pelos quais os animais ou as peças histológicas coletadas no estudo foram estabelecidos previamente à execução do experimento, são: trombose da artéria ilíaca do suíno; infecção de tecidos adjacentes; óbito do animal antes do prazo estabelecido para a coleta de material para análise histopatológica; e falhas técnicas no preparo ou processamento dos tecidos.

\section{Variáveis em estudo}

As variáveis em estudo foram: 1) aterogênese em artériasilíacas desuínossubmetidosàhomocisteinemia induzida; e 2) perviedade da artéria ilíaca dos suínos com dieta normal e dieta rica em metionina.

\section{Materiais}

O s animais utilizados foram suínos da raça M acau, originados deváriasmatrizes, com idadeaproximada de 8 semanas, peso entre 15 e 25 kg, al imentação independente e fornecidos pelo mesmo produtor. A amostra utilizada neste estudo consistiu de 14 animais.

Inicialmente, os animais foram divididos aleatoriamente em dois grupos, e cada grupo foi colocado em pocilga separada. O $\mathrm{s}$ animais foram alojados em baias com água corrente ad libitum e alimentados com ração para suínos sem suplementação lipídica adicional, balanceada eadaptada para a idade. 0 estudo foi dividido em duas fases. $\mathrm{N}$ a primeira fase, os animais foram alimentados desde o desmame (em torno de 40 dias) com uma quantidade de $950 \mathrm{~g} /$ dia de ração, durante 30 dias, para alcançar o peso desejado. A ração utilizada foi à base de cereais ( $75 \%$ de milho e $25 \%$ de soja) para 0 grupo-controle (grupo C).

N ormalmente, é necessária, na dieta suína, uma quantidade de $4,6 \mathrm{~g} /$ dia de metionina + cistina $(0,48 \%$ de metionina em $950 \mathrm{~g}$ de ração) e em torno de $8 \%$ de gordura saturada presente no leite de suínos. Sendo assim, para obter-se uma homocisteinemia induzida, 0 segundo grupo recebeu a ração com adição de 3\% de metionina na dieta também por um período de 30 dias (grupo M).

A segundafaseiniciou-se 30 diasapósa primeira, na qual foram coletadas as artériasilíacas. Asartériasforam retiradas em monoblocos para a realização de exame macroscópico e anatomopatológico. O s animais foram anestesiados e submetidos à eutanásia, através de inje- 
ção intracardíaca de cloreto de potássio, conforme o protocolo do Centro de Pesquisas do H CPA.

Os exames laboratoriais solicitados foram os seguintes: colesterol total, colesterol HDL e triglicerídeos, para permitir a inferência de que, provavelmente, estes resultados não tiveram influência no desenvolvimento da aterogênese.

A dosagem da homocisteína plasmática foi feita pelo método high performance liquid chromatography (H PLC) epara comprovar a homocisteinemia induzida pela alimentação com adição de metionina.

\section{Preparo do tecido para análise morfológica}

O s tecidos retirados foram fixados com solução de aldeído fórmico a $10 \%$ e, posteriormente, conservados a $4{ }^{\circ} \mathrm{C}$ por 10 dias. Após a fixação do segmento ressecado, a artéria ilíaca foi excisada, e realizou-senova macroscopia com descrição das alterações. Então, procedeu-se à secção de uma porção de tecido de $1 \mathrm{~cm}$ de extensão das artérias ilíacas. O s segmentos foram processados, incluídos em blocos de parafina e, posterior- mente, submetidos a cortes histológicos medindo $4 \mathrm{~cm}$ de espessura. As lâminas foram preparadas e coradas com hematoxilina-eosina.

\section{Analise estatística}

A análise dos dados foi realizada através do programaSPSS for W indows, e, para comparação das médias entre os grupos, foram utilizados o teste t de Student e o teste de $M$ ann-W hitney. 0 nível de significância (P) considerado foi de $5 \%$.

\section{Resultados}

T odos os animais sobreviveram ao experimento, não ocorreram intercorrências clínicas durante 0 estudo, e não houve alterações significativas nos níveis de colesterol total, triglicerídeos e HDL nos dois grupos. $0 \mathrm{~s}$ valores dos resultados dos exames estão expressos nas T abelas 1, 2 e 3. H ouve alteração significativa, com aumento nos níveis de homocisteína, entre o grupo $\mathrm{M}$ e o grupo $\mathrm{C}$ no período de 30 dias (T abela 4).

T abela 1 - Resultados das dosagens de colesterol total

\begin{tabular}{llccccccc}
\hline Momento & Grupo & M ediana & $\begin{array}{c}\text { Amplitude } \\
\text { interquartílica }\end{array}$ & M ínimo & Máximo & Média & $\begin{array}{c}\text { D esvio } \\
\text { padrão }\end{array}$ & $\begin{array}{c}\text { Valor } \\
\text { de P }\end{array}$ \\
\hline \multirow{2}{*}{0 dia } & Controle & 86 & 13 & 71 & 92 & 82,57 & 7,66 & 0,9015 \\
& M etionina & 85 & 6 & 63 & 111 & 85,86 & 14,01 & \\
\multirow{2}{*}{30 dias } & Controle & 85 & 31 & 71 & 120 & 90,57 & 17,92 & 0,6200 \\
& M etionina & 92 & 42 & 49 & 101 & 80,57 & 22,23 & \\
\hline
\end{tabular}

T abela 2 - Resultados das dosagens de H D L

\begin{tabular}{llccccccc}
\hline Momento & G rupo & M ediana & $\begin{array}{c}\text { Amplitude } \\
\text { interquartílica }\end{array}$ & M ínimo & Máximo & M édia & $\begin{array}{c}\text { D esvio } \\
\text { padrão }\end{array}$ & $\begin{array}{c}\text { Valor } \\
\text { de P }\end{array}$ \\
\hline \multirow{2}{*}{0 dia } & Controle & 38 & 5 & 31 & 45 & 37,71 & 4,54 & 0,0530 \\
& M etionina & 42 & 4 & 39 & 48 & 42,57 & 2,94 & \\
\multirow{2}{*}{30 dias } & Controle & 42 & 8 & 35 & 52 & 42,29 & 5,56 & 1 \\
& M etionina & 48 & 24 & 21 & 52 & 40,00 & 12,94 & \\
\hline
\end{tabular}


Tabela 3 - Resultados das dosagens de triglicerídeos

\begin{tabular}{llccccccc}
\hline Momento & Grupo & Mediana & $\begin{array}{c}\text { Amplitude } \\
\text { interquartílica }\end{array}$ & Mínimo & Máximo & M édia & $\begin{array}{c}\text { D esvio } \\
\text { padrão }\end{array}$ & $\begin{array}{c}\text { Valor } \\
\text { de P }\end{array}$ \\
\hline \multirow{2}{*}{0 dia } & Controle & 26 & 10 & 22 & 34 & 27,57 & 4,79 & 0,2086 \\
& M etionina & 33 & 15 & 21 & 45 & 33,71 & 8,48 & \\
\multirow{3}{*}{ dias } & Controle & 33 & 29 & 25 & 62 & 38,00 & 14,85 & 0,1282 \\
& M etionina & 57 & 68 & 19 & 118 & 62,29 & 36,65 & \\
\hline
\end{tabular}

T abela 4 - Resultados das dosagens de homocisteína

\begin{tabular}{llccccccc}
\hline Momento & G rupo & M ediana & $\begin{array}{c}\text { Amplitude } \\
\text { interquartílica }\end{array}$ & M ínimo & M áximo & M édia & $\begin{array}{c}\text { D esvio } \\
\text { padrão }\end{array}$ & $\begin{array}{c}\text { Valor } \\
\text { de P }\end{array}$ \\
\hline \multirow{2}{*}{0 dia } & Controle & 13,1 & 3,2 & 12 & 20 & 14,40 & 2,79 & 0,3829 \\
& M etionina & 16,4 & 10,1 & 10 & 28 & 17,47 & 6,14 & \\
\multirow{2}{*}{30 dias } & Controle & 16,1 & 5,4 & 13 & 24,2 & 16,73 & 3,88 & 0,0006 \\
& M etionina & 56,7 & 13,7 & 45 & 84,1 & 59,80 & 12,64 & \\
\hline
\end{tabular}

Por não existirem níveisnormais estabelecidospara animais de experimentação, considerou-se como normal a média dos níveis plasmáticos da homocisteína do grupo C , paraserem comparados aos níveis plasmáticos da homocisteína do grupo M . N o grupo C, não houve alteração nos níveis de homocisteína durante o experimento; os animais apresentavam valores médios de $14,40 \mu \mathrm{mol} / \mathrm{I}$ na primeira amostra e de $16,73 \mu \mathrm{mol} / \mathrm{l}$ na segunda amostra. No grupo $M$, houve aumento dos níveisséricosdahomocisteína, partindo de17,47 umol/l na primeira coleta para o valor médio de 59,80 $\mu \mathrm{mol} / \mathrm{l}$ na segunda amostra.

No exame histológico da artéria ilíaca do grupo C, predomina camada íntima delgada sem depósitos subendoteliais, lâmina elástica interna bem definida e ausência de células inflamatórias (Figura 1). N 0 grupo $M$, os cortes histológicos mostram depósitos subendoteliais de macrófagos espumosos, fazendo protusão na luz do vaso, sem sinais da presença de cálcio ou cristais de colesterol, diferentemente de lesões arteriais produzidas por dislipidemia ou a ela relacionadas, confirmando aterogênese produzida pela hiper-homocisteinemia (Figuras 2 e 3).

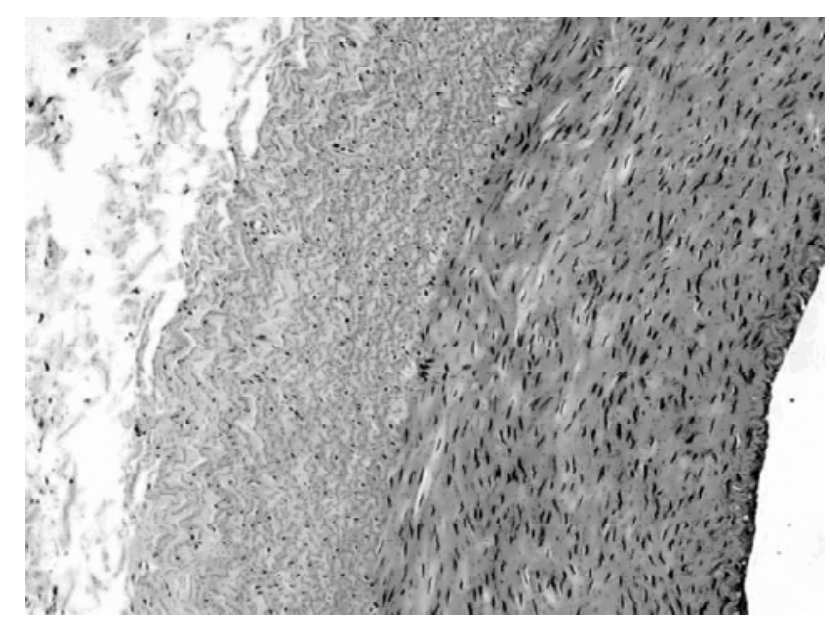

Figura 1 - Artéria ilíaca do grupo C 


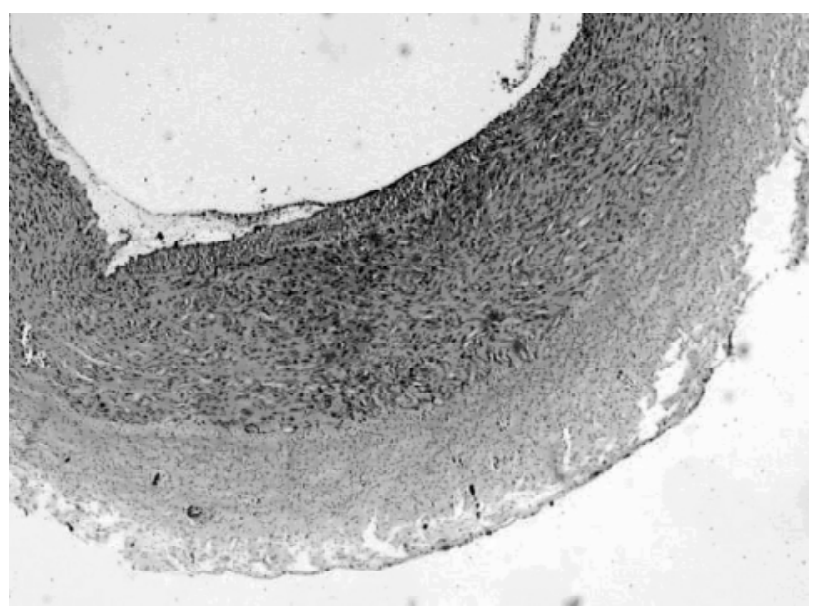

Figura 2 - Artéria ilíaca do grupo M (aumento de 40x)

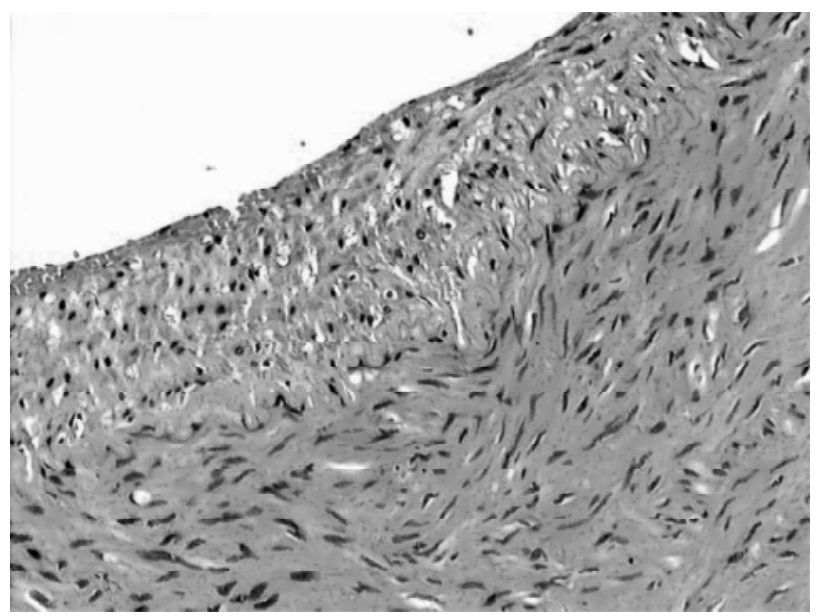

Figura 3 - Artéria ilíaca do grupo M (aumento de 200x)

\section{D iscussão}

Estudos experimentais têm demonstrado o efeito de certas substâncias químicas no desenvolvimento da aterosclerose, sugerindo que as respostas dependem da espécie do animal envolvido no estudo. Em 1974, $\mathrm{H}$ arker infundiu continuamente L-homocisteína durante 5 dias em babuínos, produzindo descamação em placas do endotélio e diminuição do tempo de sobrevida das plaquetas. Assim, concluiu que a formação de trombo arterial em pacientes homocistinúricos resulta da lesão arterial produzida pela homocisteína ${ }^{5}$.

Os resultados com experimentos a respeito do metabolismo do ácido aminossulfúrico em suínos são mais semelhantes aos obtidos com humanos. Em 1995, Rolland observou concentrações el evadas de homocisteína plasmática, lesões na lâmina elástica das artérias e ocorrência de eventos trombóticos em suínos que receberam dieta rica em metionina por 4 meses $^{6}$. Em animais hiper-homocisteinêmicos, a ativação da el astase da célula aórtica explica a degeneração característica da elastina em placas ateroscleróticas.

Recentemente, $C$ hen demonstrou que, em culturas de células do músculo liso da parede arterial de seres humanos e de carótidas de suínos, a homocisteína tem efeito prejudicial na proliferação dascélulas do músculo liso e efeito citotóxico nas células endoteliais ${ }^{7}$. Stead verificou que, colocando metionina em contato com hepatócitos de ratos, estas células transportavam mais homocisteína, sugerindo que o fígado é o responsável pela regulação dos níveis plasmáticos da mesma ${ }^{8}$. 0 efeito da homocisteína elevada no plasma de ratos diminui a vasodilatação e contribui para inativar 0 óxido nítrico no endotélio, como foi demonstrado por Eberhardt 9 .

T sai relatou que o efeito promotor do crescimento da homocisteína sobre células do músculo liso vascular, junto com seu efeito inibidor sobre o crescimento das células endoteliais, representa um mecanismo importante para elucidar a aterosclerose produzida pela homocisteína ${ }^{10}$. W oo demonstrou essefato usando ultrasom de alta resolução, o que induziu alterações no endotélio arterial queforam precursoras de aterosclerose precoceem pacientes adultosassintomáticos, porém, com homocisteína entre 34,8 $\pm 8,5 \mu \mathrm{mol} / \mathrm{I}^{11}$.

Clarke, em 1991, concluiu quea hiper-homocisteinemia é fator de risco independente para a doença vascular, incluindo coronariopatia ${ }^{12}$. 0 risco associado à hiper-homocisteinemia depende da sua concentração plasmática, e não da sua associação aos fatores de risco tradicionaisparaaaterosclerose, sendo estimado em 1,4 vezes para cada $5 \mu \mathrm{mol} / \mathrm{l}$ de aumento do total da homocisteína plasmática ${ }^{13}$.

D iversos mecanismos explicam os possíveis efeitos aterogênicos da homocisteína. É descrito o efeito citotóxico direto da homocisteína sobre as células endoteliais e, possivelmente, sobre a síntese de prostaciclina. Tem sido sugerido efeito sobre as plaquetas, aumentando sua adesividade e agregabilidade, favorecendo a trombose. Além disso, al gunsestudostambém demonstraram efeitos sobre fatores da coagulação, favorecendo a trombofilia com ativação do fator $\mathrm{V}^{14}$. 
Ao administrar, em coelhos, uma dieta com $2 \%$ de colesterol e, em outro grupo, a mesma dieta, mas com ausência de vitamina $B_{6}$ e injetando homocisteína subcutânea, M cC ully demonstrou lesões ateroscleróticas mais inten sas no grupo no qual foi injetado homocisteína ${ }^{15}$. Recentemente, Sthalkemostrou aterogênese em aorta decoel hosao adicionar metioninaà dieta ${ }^{16} .0$ presentetrabal ho demonstraum model o deaterogênese em suínos num período relativamente curto (30 dias), mediante homocisteinemia induzida pela ingestão oral de metionina.

\section{Conclusão}

Concluiu-se que a homocisteinemia induzida pela ingestão de metionina, por um período de 30 dias, causa aterogênese na artéria ilíaca de suínos.

\section{Referências}

1. Ross R. Atherosclerosis: an inflammatory disease. N Engl J M ed. 1999;340:115-26.

2. Libby $P$, Ridker PM, M aseri A. Inflammation and atherosclerosis. Circulation. 2002;105:1135-43.

3. N ehler M R, T aylor LM J r, Porter JM . H omocysteinemia as a risk factor for atherosclerosis: a review. Cardiovasc Surg. 1997;5:559-67.

4. Venancio LS, Burini RC, YoshidaW B. H iper-homocisteinemia na doença arterial periférica. J Vasc Br. 2004;3:31-7.

5. H arker LA, Slichter SJ, Scott CR, Ross R. H omocystinemia: vascular injury and arterial thrombosis. N Engl J Med. 1974;291:537-43.

6. Rolland PH, Friggi A, Barlatier A, et al. Hyperhomocysteinemia-induced vascular damage in the minipig. Captopril-hydrochlorothiazide combination prevents elastic alterations. Circulation. 1995;91:1161-74.
7. Chen $\mathrm{C}, \mathrm{H}$ alkos $\mathrm{M} \mathrm{E}$, Surowiec $\mathrm{SM}$, Conklin BS, Lin PH, Lumsden $A B$. Effects of homocysteine on smooth muscle cell proliferation in both cell culture and artery perfusion culture models. J Surg Res. 2000;88:26-33.

8. Stead LM, Brosnan ME, Brosnan JT. Characterization of homocysteine metabolism in the rat liver. Biochem $\mathrm{J}$. 2000;350:685-92.

9. Ebenhardt RT, Forgione MA, Cap A, et al. Endothelial dysfunction in a murinemodel of mild hyperhomocysteinemia. J Clin Invest. 2000;106:483-91.

10. T sai JC, Perrella MA, Yoshizumi M, et al. Promotion of vascular smooth muscle cell growth by homocysteine: alink to atherosclerosis. Proc N atl Acad Sci U SA. 1994;91:6369-73.

11. W oo KS, Chook P, Lolin YI, et al. H yperhomocyst(e)inemia is a risk factor for arterial endothelial dysfunction in humans. Circulation. 1997;96:2542-4.

12. ClarkeR, D aly L, Robinson $K$, et al. H yperhomocysteinemia: an independent risk factor for vascular disease. N Engl J M ed. 1991;324:1149-55.

13. W ilcken $D E, W$ ilcken $B$. T he pathogenesis of coronary artery disease. A possible role for methionine metabolism. J Clin Invest. 1976;57:1079-82.

14. den Heijer $M$, Koster $T$, Blom $H J$, et al. $\mathrm{H}$ yperhomocysteinemia as a risk factor for deep-vein thrombosis. N Engl J M ed. 1996;334:759-62.

15. M cC ully KS, Ragsdale BD. Production of arteriosclerosis by homocysteinemia. Am J Pathol. 1970;61:1-11.

16. Stahlke Jr HJ, França LH G, StahlkePH, StahlkePS. H iperhomocisteinemia causando aterogênese na aorta de coel hos modelo experimental. J Vasc Br. 2004;3:20-30.

\author{
Correspondência: \\ Luís H enrique Gil França \\ Rua C oronel D ulcídio, 1189/1801 \\ CEP 80250-100 - Curitiba, PR \\ Tel.: (41) 3343.0963 \\ E-mail: luishgf@hotmail.com
}

